\title{
Studies on Enhancement of Anti-microbial Activity of Pristine MWCNTs Against Pathogens
}

\author{
Shikha Lohan, ${ }^{1}$ Kaisar Raza, ${ }^{1,2,5}$ Saloni Singla, ${ }^{3}$ Sanjay Chhibber, ${ }^{3}$ Sheetu Wadhwa, ${ }^{4}$ O. P. Katare, ${ }^{4}$ \\ Pramod Kumar, ${ }^{2}$ and Bhupinder Singh ${ }^{1,4,5}$
}

Received 4 April 2015; accepted 8 October 2015; published online 22 October 2015

\begin{abstract}
Carbon nanotubes (CNTs), owing to their inherently unique properties in the domain of biomedical sciences including drug delivery, offer an exciting platform to the researchers. Of late, their applications have also been successfully established. Recently, single-walled CNTs (SWCNTs) have been explored for antibacterial efficacy, but naïve multi-walled CNTs (MWCNTs) still remained unearthed. The present studies endeavor the investigation of the potential of various non-ionic surfactants in solubility enhancement of MWCNTs and their subsequent antibacterial efficacy against Escherichia coli and Staphylococcus aureus. Polysorbates offer more solubility to MWCNTs vis-à-vis the phospholipids. However, the antibacterial effect was found to be less influenced by solubility but significantly determined by the type of surfactant. Transmission electron photomicrographs confirmed significant adhesion of MWCNTs to the bacterial walls only in the presence of unsaturated phospholipids and this was expressed in the form of lowest minimum inhibitory concentration (MIC) values of MWCNTs dispersed with the same. The findings are unique as MWCNTs were found to be active against both Gram-negative and Gram-positive bacteria to a similar extent, though somewhat milder than SWCNTs. However, when dispersed with unsaturated phospholipids, the former offer almost comparable antibacterial effects to that of the latter. The study opens a new research domain to further explore the antibacterial effects of non-functionalized and relatively safer MWCNTs, accentuating the importance of biocomponents like unsaturated phospholipids in this purview.
\end{abstract}

KEY WORDS: antibacterial; dispersability; Escherichia coli and Staphylococcus aureus; phospholipid; polysorbate.

\section{INTRODUCTION}

Carbon nanotubes (CNTs) have been an exciting research domain since their discovery in 1991 in the form of small by-product of fullerene synthesis (1). Owing to their unique mechanical, physical, thermal, and chemical properties, CNTs are being widely explored for their applications in diverse domains including drug delivery (2,3). Lately, a divergence towards CNTs has been utilized in scientific circles, as drug delivery carriers, as a few recent research reports indicate their superiority over the routinely

\footnotetext{
${ }^{1}$ UGC-Centre of Excellence in Applications of Nanomaterials, Nanoparticles and Nanocomposites (Biomedical Sciences), Panjab University, Chandigarh, India 160014.

${ }^{2}$ Department of Pharmacy, School of Chemical Sciences and Pharmacy, Central University of Rajasthan, Bandar Sindri, Distt. Ajmer, Rajasthan, India 305817.

${ }^{3}$ Department of Microbiology, Panjab University, Chandigarh, India 160014.

${ }^{4}$ Department of Pharmaceutics, University Institute of Pharmaceutical Sciences, Panjab University, Chandigarh, India 160014.

${ }^{5}$ To whom correspondence should be addressed. (e-mail: drkaisar@curaj.ac.in, bsbhoop@yahoo.com)
}

employed colloidal drug delivery carriers, viz. liposomes, nanoparticles, and dendrimers (4). In most of the studies, conducted so far, CNTs have been employed as "cargo vehicles" to deliver the drug to the desired sites $(3,4)$. The naïve CNTs also possess noticeable antimicrobial action, though the reports are sparse (5). The recent findings on CNTs are encouraging and are acting as a driving force for further research on these so-called "inorganic cargo vehicles" (4).

The present era of nanotechnology marks increased attention towards the biocompatibility and toxicity issues of the nanoscale drug delivery carriers. CNTs, in this context, offer substantial biocompatibility, esp. MWCNTs in comparison to single-walled CNTs (SWCNTs), which get further increases with enhancement in aqueous dispersability $(3,6,7)$. Several approaches, including physical and chemical methods, are being employed to enhance the aqueous dispersability of CNTs to increase the biological acceptance of these carriers (3). One such approach is to coat the CNTs with hydrophilic surfactants. A lot many studies, in this regard, have been reported in literature employing surfactants like sodium dodecylbenzenesulfonate (8), dodecyltrimethylammonium bromide (9), hexadecyltrimethylammonium bromide (10), octylphenol 
ethoxylate (Triton X-100) (11), sodium dodecyl sulfate (SDS) $(12,13)$, and polysorbates (13).

Triton X-100 has been reported to offer maximum dispersability vis-à-vis Tween 20, Tween 80, and SDS (13), though Triton X-100 is reported to be relatively more toxic than polysorbates (14). A few scattered reports are also focused on the use of phospholipids as CNT-dispersing agents (15-17).

Antimicrobial activity of CNTs was first reported in 2007 (18). Since then, numerous studies have been published concentrating on antimicrobial effects of CNTs and correlating it with CNT dimensions (19), functionalization (20), aggregation, concentration, contact time, and intensity (21-23). Most of the antimicrobial studies have been focused on SWCNTs (24), while MWCNTs are little explored, that too with chemical modifications $(20,25,26)$. Hardly any publication deals with the concentration-dependent antimicrobial activity of non-functionalized MWCNTs employing biocompatible surfactants viz. variety of phospholipids and polysorbates.

The present study explores the effect of various phospholipids (Phospholipon S 100, Phospholipon 85 G, Phospholipon $90 \mathrm{G}$, and Phospholipon $90 \mathrm{NG}$ ) and polysorbates (Tween 20, Tween 40, and Tween 80 ) on the solubility of naïve MWCNTs and subsequent antimicrobial activity against Escherichia coli and Staphylococcus aureus. The current work not only attempts to determine the solubilization capacity of biocompatible surfactants but also establishes a relationship between the extent of solubilization and antibacterial activity.

\section{MATERIALS}

Lyophilized spores of E. coli (MTCC No: 40) and S. aureus (MTCC No: 3160) were procured from The Microbial Type Culture Collection and Gene Bank, Institute of Microbial Technology (IMTECH), Chandigarh. A variety of phospholipids (Phospholipon S $100 \AA$, Phospholipon $85 \mathrm{G} \AA$, Phospholipon $90 \mathrm{G} \AA$, and Phospholipon $90 \mathrm{NG}^{\circledR}$ ) were obtained ex-gratis from M/s Phospholipid GmbH, Nattermannallee, Germany. MWNTs ( $>95 \%$ purity, L-0.5-2 $\mu \mathrm{m}, \mathrm{D}-20-50 \mathrm{~nm}$ ) were purchased from M/s Nanostructured and Amorphous Materials Inc., USA. Polysorbates (Tween 20, Tween 40, and Tween 80) were procured from M/s Fisher Scientific, Mumbai, India. All other chemicals employed in various studies were of analytical grade and were used as such. Ultrapure water (Milli-Q ${ }^{\circledR}$ Integral system; M/s Merck Millipore, Billerica, USA) was used throughout the studies.

\section{METHODS}

\section{Solubility Studies}

To each eppendorf tube of $2 \mathrm{~mL}$ capacity, amounts of 4,10 , and $40 \mathrm{mg}$ of various phospholipids and polysorbates were added individually. After adding $1 \mathrm{~mL}$ of phosphate-buffered saline, pH 7.4 (PBS), MWCNTs
(1 mg) were added to each eppendorf tube. To facilitate dispersion of the surfactants in PBS, the eppendorf tubes were vortexed and subsequently sonicated in a water bath for $1 \mathrm{~h}$. The mixtures were centrifuged for $3 \mathrm{~min}$. at $12,320 \mathrm{~g}$ relative centrifugal force. The clear supernatant was transferred to a cuvette and read for absorbance at $360 \mathrm{~nm}$ against the corresponding blank using a UVvisible spectrophotometer. The results were reported in terms of solubility enhancement, which was further determined as the ratio of optical density (OD) of the test to the OD of MWCNTs without phospholipid/polysorbate in PBS (16).

\section{Antibacterial Studies}

The minimum inhibitory concentration (MIC) of each MWCNT set was determined as per the Clinical and Laboratory Standards Institute (CLSI) guidelines (27). A single colony of $E$. coli and $S$. aureus was inoculated in $10 \mathrm{~mL}$ of Tryptone Soya Broth (TSB) and incubated overnight at $37^{\circ} \mathrm{C}$. Next day, an aliquot of $10 \mu \mathrm{L}$ of the overnight culture was inoculated in $10 \mathrm{~mL}$ of TSB and incubated till $\mathrm{OD}_{600}$ of bacterial culture reached 0.1 corresponding to a cell density of $10^{7} \mathrm{CFU} / \mathrm{mL}$. To ten different glass tubes in three sets, $1.0 \mathrm{~mL}$ of TSB was added. To the first tube of each set containing $1.0 \mathrm{~mL}$ of TSB, $1.0 \mathrm{~mL}$ of the MWCNTs stock solution was added. Serial dilutions were made by transferring $1.0 \mathrm{~mL}$ from the first tube to the last tube serially in all the three sets. Then, $100 \mu \mathrm{L}$ of bacterial culture $\left(10^{7} \mathrm{CFU} / \mathrm{mL}\right)$ was added to the tubes containing different concentrations of MWCNTs. MWCNT sterility control check was done for all the samples in which only MWCNTs were added to the growth medium. Another set of tubes containing TSB and bacterial culture was employed to test the growth supporting property of the medium and served as another control. Thereafter, all test and control tubes were incubated overnight at $37^{\circ} \mathrm{C}$. The lowest concentration of MWCNTs that prevented the appearance of visible turbidity after overnight incubation was taken as the MIC. The particle size and zeta potential values of various dispersions were also studied using a Zeta Sizer (Malvern Zetasizer Nano ZS 90, UK).

\section{MTT Assay}

In order to determine unwanted cytotoxicity on mammalian cells, MTT assay (3-(4, 5-dimethylthiazol-2-yl)-2,5diphenyltetrazolium bromide, a tetrazole) was carried out as per already established protocol on non-invasive MCF7 cells and invasive MDB-MA-231 cells. The cells were seeded in a 96-well plate in RPMI-1640 culture medium containing $10 \% \mathrm{FBS}$ and incubated for $24 \mathrm{~h}$ at $37^{\circ} \mathrm{C}$ and $5 \% \mathrm{CO}_{2}$ humidified incubator. After $24 \mathrm{~h}$, cells were attached to the well plate and exposed to MWNTs at different concentrations and incubated for another $24 \mathrm{~h}$. After $24 \mathrm{~h}, 10 \mathrm{~mL}$ of MTT $\left(5 \mathrm{mg} \mathrm{mL}^{-1}\right)$ prepared in RPMI-1640 was added to each well and incubated for $4 \mathrm{~h}$ (28). After incubation, $100 \mathrm{~mL}$ of media was 
discarded from each well and $100 \mathrm{~mL}$ of DMSO, a solubilizing agent, was added to each well to dissolve the formazan crystal inside the cells which was then shaken at ELISA shaker for $30 \mathrm{~s}$ and absorbance was measured at $570 \mathrm{~nm}$.

\section{Morphology of the Dispersed MWCNTs}

Morphology of the MWCNTs showing antibacterial activity was determined with the aid of transmission electron microscope (TEM) installed at Central Instrumentation Laboratory, Panjab University, Chandigarh, India. A 10$\mu \mathrm{L}$ drop of the samples, viz. MWCNT suspension and MWCNT along with E. coli suspension, was placed on to carbon-coated grid, viewed and photographed under the electron microscope at suitable magnification. The TEM microphotographic images of MWCNTs, before and after incubation in phospholipids, were also obtained.

\section{RESULTS AND DISCUSSION}

\section{Solubility Studies}

Figure 1 shows the enhancement in the solubility of MWCNTs by virtue of various phospholipids and polysorbates. In general, the effect of polysorbates was found to be more pronounced than that of phospholipids. It might be plausibly due to the relatively higher lipophilicity and less water dispersability of the phospholipids in water. Phospholipids have very little aqueous solubility of the order of $\leq 10^{-8} \mathrm{M}$, while other detergents like polysorbates have the aqueous solubility in the range from $10^{-4}$ to $10^{-2} \mathrm{M}$
(29). However, the trends obtained in the solubility enhancement were different at various concentrations explored. This difference in the trend cannot be ascribed to the variation in the critical micelle concentrations (CMCs), as all the concentrations selected were above the CMC values of phospholipids $(\sim 0.1 \mathrm{mM})$ and polysorbates ( 0.01-0.06 mM) (30-32). The conceivable reason for such results can be the difference of the non-polar chain of the various surfactants, resulting in a specific concentrationdependent steric orientation (13).

At moderate and higher concentrations, the dispersing and solubilization capabilities of Tween 20 were observed to be much more prominent vis-à-vis Tween 80 , contrary to the observations reported in the literature (13). This experimentally observed trend can be attributed to the chemical nature of the polysorbates, as the hydrocarbon chain length and the adsorption of a surfactant on the CNTs invariably show an inverse relationship. Tween 80 has relatively longer hydrocarbon chain vis-à-vis Tween 20 and hence is expected to show less adsorption and subsequently inferior dispersion capabilities (33).

\section{Antibacterial Studies}

Despite marked solubility enhancement, the CNTs dispersed in polysorbates offered milder antibacterial efficacy than that of those dispersed with the unsaturated phospholipid (Phospholipon 90 G). MWCNTs dispersed in other phospholipids and polysorbates offered higher values of MIC than the MWCNTs dispersed by virtue of unsaturated phospholipid. The MIC offered by the

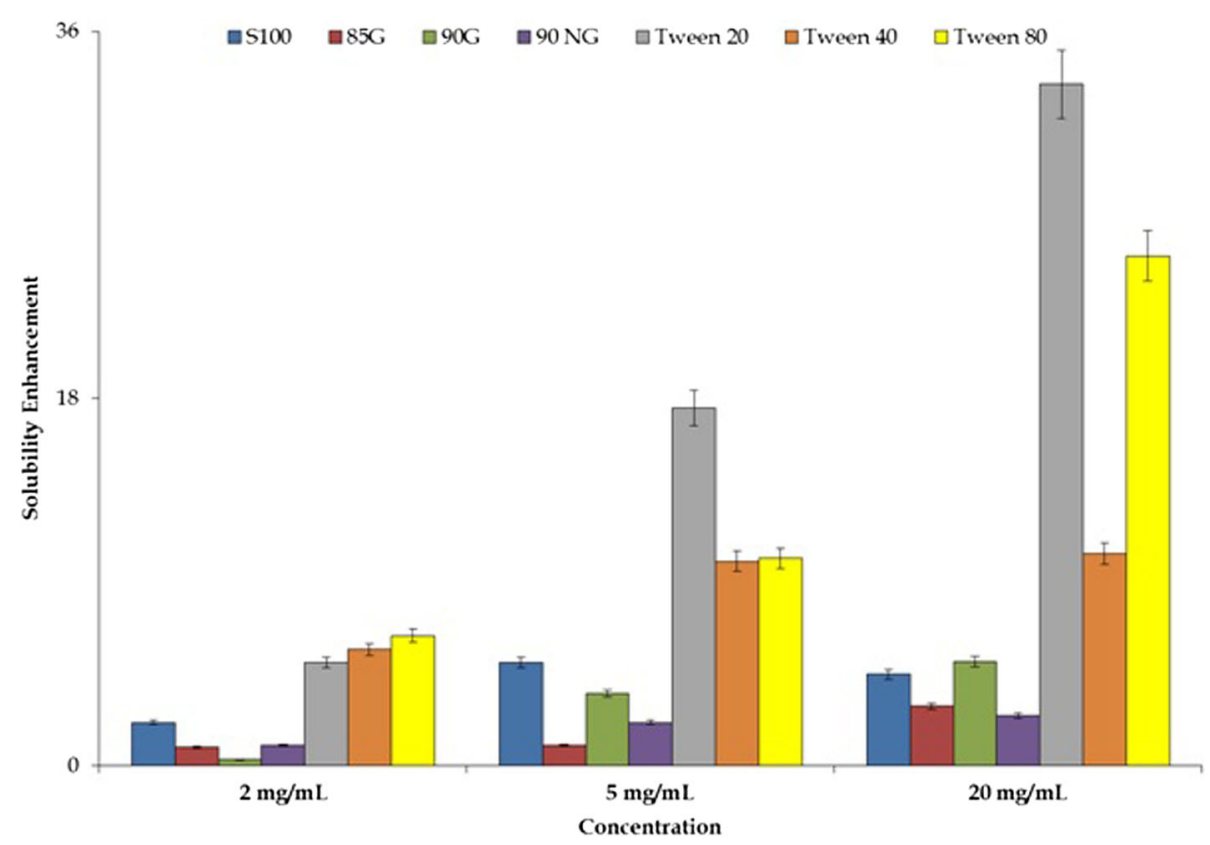

Fig. 1. Solubility enhancement of MWCNTs in PBS at various concentrations of phospholipids and polysorbates $(n=3)$. Each crossbar indicated \pm 1 SD 
MWCNTs dispersed by virtue of the unsaturated phospholipid was found to be $0.5 \mathrm{mg} / \mathrm{mL}$, while other dispersed MWCNTs expressed MIC value of $2 \mathrm{mg} / \mathrm{mL}$. However, the blank surfactants and phospholipids did not induce any noticeable antimicrobial activity in the studied concentrations. The findings are in consonance with the earlier reports of enhancement of antimicrobial efficacy with the systems employing unsaturated phospholipids but for other colloidal carriers $(34,35)$. These biomembrane components, i.e., unsaturated phospholipids, facilitate the adherence of the nanosystems with the bacterial cell walls, resulting in the enhancement of the contact time and ultimately the bactericidal action (23). The obtained values of MIC are a bit higher than that observed with SMWCNTs and $\mathrm{C}_{60}$ fullerenes, thus indicating relatively less antimicrobial potential of MWCNTs than these carbon-based nanostructures (19,36). However, the present studies unequivocally construed that when dispersed in unsaturated phospholipids, MWCNTs can offer equivalent antibacterial effect to SWCNTs and fullerenes.

Particle size of various MWCNT dispersions showed a homogenous trend with size ranging between 870 and $1300 \mathrm{~nm}$, in consonance with already published results (37). In addition, the zeta potential values of Phospholipid $90 \mathrm{G}$ (i.e., an unsaturated phospholipid) ranged between -4.17 and $-11.3 \mathrm{mV}$, augmenting significantly with increasing concentration of the phospholipid. Interestingly, on the other hand, the magnitudes of zeta potential for saturated phospholipids (ranging between -10.99 and $-17.11 \mathrm{mV}$ ) and other studied surfactants (ranging between -10.94 to $-20.90 \mathrm{Mv}$ ) were relatively higher, also exhibiting an increasing trend with increasing concentrations. The zeta potential of blank CNT dispersion was observed to be $-10.80 \mathrm{mV}$ (Fig. 2). These negative values of zeta potential of MWCNT dispersions are in consonance with the previous published reports $(38,39)$. Besides the cell-puncturing potential of MWCNTs, increased adhesion of these carbon-based nanoconstructs can be ascribed to the involvement of electrostatic factors too (40). The lower negative charge intensity on MWCNTs might have accounted for reduced repulsion by the negatively surfaced bacterial walls, resulting in relatively better adhesion of the MWCNTs and, thus, improved antibacterial efficacy.

\section{MTT ASSAY}

Cells were exposed to MWNTs at two different concentrations, i.e., 1 and $10 \mu \mathrm{g} / \mathrm{mL}$, and absorbance was calculated at $570 \mathrm{~nm}$ on ELISA reader when exposed to noninvasive MCF-7 cells and invasive MDB-MA-231 cells. The graph represented in Fig. 3 is plotted between cytotoxicity percentage and concentration of two different treated cell lines with MWNTs (41). It clearly vouches the non-toxic nature of the studied carbon-based nanoconstructs on human cells.

\section{Morphology of the Dispersed MWCNTs}

As is vivid from Fig. 4, the CNTs dispersed in polysorbate 80 show relatively less adsorption on to the surface of $E$. coli vis-à-vis the CNTs dispersed in unsaturated phospholipid. These findings support the results obtained in the antibacterial studies indicating the enhanced bacterial adsorption by virtue of unsaturated phospholipids (35). The results are unique in nature and provide the first instance of the bacterium cell completely surrounded by MWCNTs when dispersed with the aid of biocomponents like unsaturated phospholipids. However, polysorbates (detergents) also aid in a bit of adsorption but not as strong as offered by Phospholipon $90 \mathrm{G}$.

TEM images in Fig. 5 clearly depict the role of polysorbate 80 in dispersing the bundles of MWCNTs as compared to these before dispersing them in surfactants.

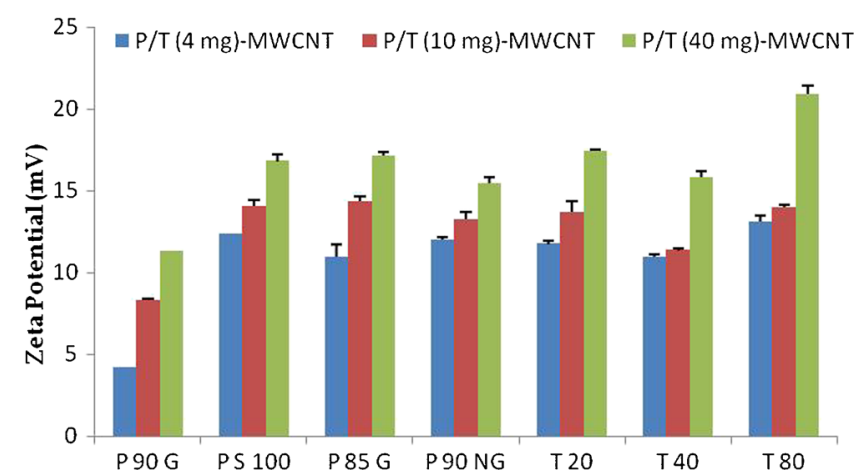

Fig. 2. Zeta potential values for MWCNT dispersions with phospholipids (saturated/unsaturated) and polysorbates $(n=3)$. Each crossbar indicates $\pm 1 \mathrm{SD}$, where $P$ stands for phospholipids and $T$ for tweens (polysorbates)

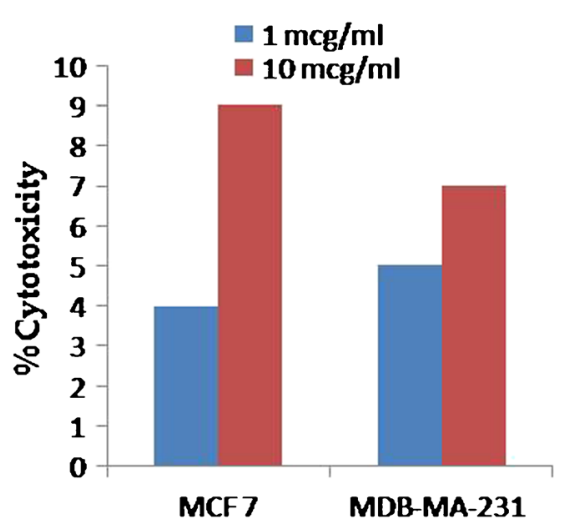

Fig. 3. Graph between cytotoxicity percentage on two different cell lines when incubated with MWCNTs 

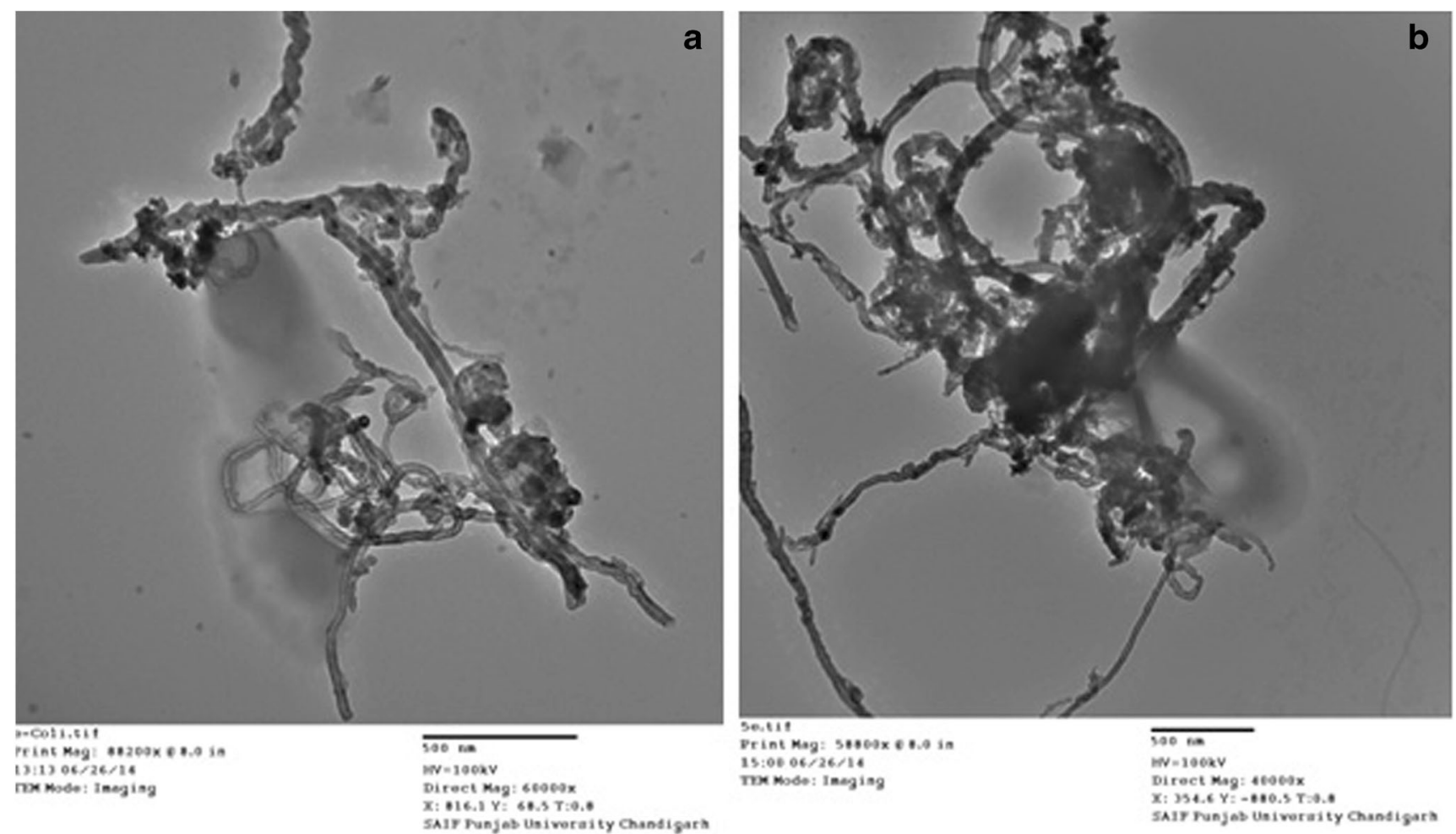

Fig. 4. TEM images of MWCNTs incubated with E. coli after being dispersed in: (a) polysorbate 80; and (b) Phospholipon 90
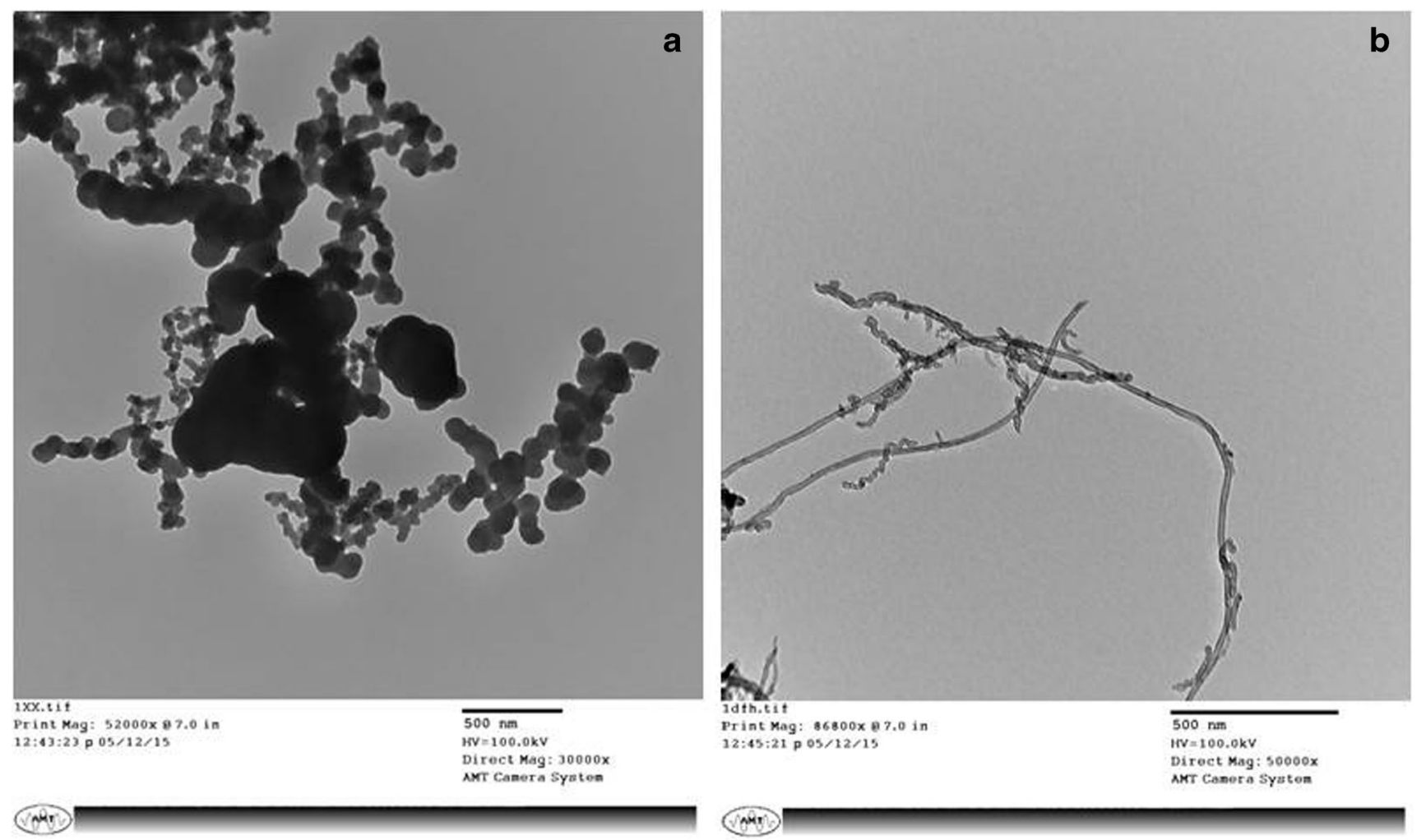

Fig. 5. TEM images of MWCNTs incubated: (a) before; and (b) after dispersing the CNT in various polysorbate 80 


\section{CONCLUSIONS}

The present studies prove the potential of non-ionic surfactants including phospholipids in enhancing the aqueous solubility of MWCNTs, indicating the marked difference in solubility offered by various polysorbates as well as phospholipids. Interestingly, MWCNTs offered significant antimicrobial effect against both Gram-negative (E. coli) and Grampositive ( $S$. aureus) bacteria, though somewhat milder than the reported results of SWCNTs and fullerenes. However, the findings reported here clearly demonstrate the enhanced adsorption of MWCNTs on the bacterial walls, resulting in MIC values of MWCNTs comparable to the established strong antimicrobials viz., SWCNTs and fullerenes. The studies provide a platform to explore the potential of relatively safer CNTs, i.e., MWCNTs, in the domain of antimicrobials.

\section{ACKNOWLEDGMENTS}

The authors acknowledge the financial support provided by University Grants Commission, New Delhi, India to the Centre of Excellence in Application of Nanomaterials, Nanoparticles and Nanocomposites (Biomedical Sciences), Panjab University, Chandigarh. Authors also acknowledge the timely advice and help of Ms Shweta Arora, Central Scientific Instruments Organization, Chandigarh and the generosity of $\mathrm{M} / \mathrm{s}$ Phospholipid GmbH, Nattermannallee, Germany for the ex-gratis supply of various phospholipids.

\section{REFERENCES}

1. Hamada N, Sawada S-I, Oshiyama A. New one-dimensional conductors: graphitic microtubules. Phys Rev Lett. 1992;68(10):1579.

2. Liu Z, Sun X, Nakayama-Ratchford N, Dai H. Supramolecular chemistry on water-soluble carbon nanotubes for drug loading and delivery. ACS Nano. 2007;1(1):50-6.

3. Arora S, Saharan R, Kaur H, Kaur I, Bubber P, Bharadwaj LM. Attachment of docetaxel to multiwalled carbon nanotubes for drug Delivery applications. Adv Sci Lett. 2012;17(1):70-5.

4. Mody N, Tekade RK, Mehra NK, Chopdey P, Jain NK. Dendrimer, liposomes, carbon nanotubes and PLGA nanoparticles: one platform assessment of drug delivery potential. AAPS PharmScitech. 2014;15(2):388-99.

5. Olivi M, Zanni E, De Bellis G, Talora C, Sarto MS, Palleschi C, et al. Inhibition of microbial growth by carbon nanotube networks. Nanoscale. 2013;5(19):9023-9.

6. Schrand AM, Dai L, Schlager JJ, Hussain SM, Osawa E. Differential biocompatibility of carbon nanotubes and nanodiamonds. Diam Relat Mater. 2007;16(12):2118-23.

7. Li X, Wang L, Fan Y, Feng Q, Cui F-z. Biocompatibility and toxicity of nanoparticles and nanotubes. J Nanomater. 2012;2012:6.

8. Islam M, Rojas E, Bergey D, Johnson A, Yodh A. High weight fraction surfactant solubilization of single-wall carbon nanotubes in water. Nano Lett. 2003;3(2):269-73.

9. Whitsitt EA, Barron AR. Silica coated single walled carbon nanotubes. Nano Lett. 2003;3(6):775-8.

10. Ryabenko A, Dorofeeva T, Zvereva G. UV-VIS-NIR spectroscopy study of sensitivity of single-wall carbon nanotubes to chemical processing and Van-der-Waals SWNT/SWNT interaction. Verification of the SWNT content measurements by absorption spectroscopy. Carbon. 2004;42(8):1523-35.

11. Wang H, Zhou W, Ho DL, Winey KI, Fischer JE, Glinka CJ, et al. Dispersing single-walled carbon nanotubes with surfactants: a small angle neutron scattering study. Nano Lett. 2004;4(9):1789-93.

12. Yu J, Grossiord N, Koning CE, Loos J. Controlling the dispersion of multi-wall carbon nanotubes in aqueous surfactant solution. Carbon. 2007;45(3):618-23.

13. Rastogi R, Kaushal R, Tripathi S, Sharma AL, Kaur I, Bharadwaj LM. Comparative study of carbon nanotube dispersion using surfactants. J Colloid Interface Sci. 2008;328(2):421-8.

14. Oberle R, Moore T, Krummel D. Evaluation of mucosal damage of surfactants in rat jejunum and colon. J Pharmacol Toxicol Methods. 1995;33(2):75-81.

15. Richard C, Balavoine F, Schultz P, Ebbesen TW, Mioskowski C. Supramolecular self-assembly of lipid derivatives on carbon nanotubes. Science. 2003;300(5620):775-8.

16. Wu Y, Hudson JS, Lu Q, Moore JM, Mount AS, Rao AM, et al. Coating single-walled carbon nanotubes with phospholipids. J Phys Chem B. 2006;110(6):2475-8.

17. Douroumis D, Fatouros DG, Bouropoulos N, Papagelis K, Tasis D. Colloidal stability of carbon nanotubes in an aqueous dispersion of phospholipid. Int J Nanomedicine. 2007;2(4):761.

18. Kang S, Pinault M, Pfefferle LD, Elimelech M. Single-walled carbon nanotubes exhibit strong antimicrobial activity. Langmuir. 2007;23(17):8670-3.

19. Kang S, Herzberg M, Rodrigues DF, Elimelech M. Antibacterial effects of carbon nanotubes: size does matter! Langmuir. 2008;24(13):6409-13.

20. Zardini HZ, Amiri A, Shanbedi M, Maghrebi M, Baniadam M. Enhanced antibacterial activity of amino acids-functionalized multi walled carbon nanotubes by a simple method. Colloids Surf B: Biointerfaces. 2012;92:196-202.

21. Liu S, Wei L, Hao L, Fang N, Chang MW, Xu R, et al. Sharper and faster "nano darts" kill more bacteria: a study of antibacterial activity of individually dispersed pristine single-walled carbon nanotube. ACS Nano. 2009;3(12):3891-902.

22. Arias LR, Yang L. Inactivation of bacterial pathogens by carbon nanotubes in suspensions. Langmuir. 2009;25(5):3003-12.

23. Liu S, Ng AK, Xu R, Wei J, Tan CM, Yang Y, et al. Antibacterial action of dispersed single-walled carbon nanotubes on Escherichia coli and Bacillus subtilis investigated by atomic force microscopy. Nanoscale. 2010;2(12):2744-50.

24. Dong L, Henderson A, Field C. Antimicrobial activity of singlewalled carbon nanotubes suspended in different surfactants. J Nanotechnol. 2012;2012:7.

25. Prodana M, Ionita D, Ungureanu C, Bojin D, Demetrescu I. Enhancing antibacterial effect of multiwalled carbon nanotubes using silver nanoparticles. Dig J Nanomater Biostruct. 2011;6(2):549-56.

26. Qi X, Gunawan P, Xu R, Chang MW. Cefalexin-immobilized multi-walled carbon nanotubes show strong antimicrobial and anti-adhesion properties. Chem Eng Sci. 2012;84:552-6.

27. Wayne PA. Performance standards for antimicrobial susceptibility testing: Sixteenth informational supplement: Twenty-second informational supplement. CLSI document M100-S22. Clinical and Laboratory Standards Institute; 2012.

28. Arora S, Kumar R, Kaur H, Rayat CS, Kaur I, Arora SK, et al. Translocation and toxicity of docetaxel multi-walled carbon nanotube conjugates in mammalian breast cancer cells. J Biomed Nanotechnol. 2015;10:3601-9.

29. Buboltz JT, Feigenson GW. Phospholipid solubility determined by equilibrium distribution between surface and bulk phases. Langmuir. 2005;21(14):6296-301.

30. King MD, Marsh D. Head group and chain length dependence of phospholipid self-assembly studied by spin-label electron spin resonance. Biochemistry. 1987;26(5):1224-31.

31. Patist A, Bhagwat S, Penfield K, Aikens P, Shah D. On the measurement of critical micelle concentrations of pure and technicalgrade nonionic surfactants. J Surfactant Deterg. 2000;3(1):53-8.

32. Ćirin DM, Poša MM, Krstonošić VS, Milanović ML. Conductometric study of sodium dodecyl sulfate-nonionic surfactant (Triton X-100, Tween 20, Tween 60, Tween 80 or Tween 85) mixed micelles in aqueous solution. Hemijska Industrija. 2012;66(1):21-8.

33. Napper DH. Polymeric stabilization of colloidal dispersions. 2nd ed. London: Academic Press; 1983. 
34. Yang D, Pornpattananangkul D, Nakatsuji T, Chan M, Carson D, Huang C-M, et al. The antimicrobial activity of liposomal lauric acids against Propionibacterium acnes. Biomaterials. 2009;30(30):6035-40.

35. Raza K, Singh B, Singla S, Wadhwa S, Garg B, Chhibber S, et al. Nanocolloidal carriers of isotretinoin: antimicrobial activity against Propionibacterium acnes and dermatokinetic modeling. Mol Pharm. 2013;10(5):1958-63.

36. Tsao N, Luh T-Y, Chou C-K, Chang T-Y, Wu J-J, Liu C-C, et al. In vitro action of carboxyfullerene. J Antimicrob Chemother. 2002;49(4):641-9.

37. Rieger C, Kunhardt D, Kaufmann A, Schendel D, Huebner D, Erdmann K, et al. Characterization of different carbon nanotubes for the development of a mucoadhesive drug delivery system for intravesical treatment of bladder cancer. Int J Pharm. 2015;479(2):357-63.
38. Lee J, Kim M, Hong CK, Shim SE. Measurement of the dispersion stability of pristine and surface-modified multiwalled carbon nanotubes in various nonpolar and polar solvents. Meas Sci Technol. 2007;18:3707-12.

39. Griffith A, Notley SM. pH dependent stability of graphene suspensions with ad-sorbed weakly ionisable cationic polyelectrolyte. J Colloid Interface Sci. 2012;369:210-5.

40. Chen H, Wang B, Gao D, Guan M, Zheng L, Ouyang H, et al. Broad-spectrum antibacterial activity of carbon nanotubes to human gut bacteria. Small. 2013;9(16):273546.

41. Arora S, Kaur H, Kumar R, Kaur R, Rana D, Rayat CS, et al. In vitro cytotoxicity of multiwalled and single walled carbon nanotubes on human cell lines. Fullerenes Nanotubes Carbon Nanostruct. 2015;23(5):377-82. 